bicarbonat. Nach Zusatz von 2 bis 3 Tropfen 20-proz. Kaliumjodidlösung und ebensoviel Stärkelösung titriert man mit 0,1-n. Chloramin bis zum Auftreten einer leichten Blaufärbung.

Die Berechnung der Analyse ist am besten aus einem Beispiel ersichtlich.

Zur Analyse gelangten $60 \mathrm{ccm}$ Gas. Zugegeben wurden $20,0 \mathrm{ccm} 0,1-n$. Kaliumbromidbromat und $25,0 \mathrm{ccm}$ einer 0,1-n. Lösung von arseniger Säure. Bei der Titration wurden $11,15 \mathrm{ccm} 0,1-n$. Chloramin verbraucht. Temperatur $20^{\circ} \mathrm{C}$, Druck $750 \mathrm{~mm}$ Hg-Säule.

Aus diesen Meßdaten ergibt sich:

Zur Reduktion des überschüss. Broms wurden 25,0 $-11,75=13,25 \mathrm{ccm}$ der Lösung von arseniger Säure verbraucht. Diese Menge entspricht einer ebenso großen an Bromidbromatlösung. Zur Absorption des Äthylens wurde also so viel Brom verbraucht, wie $20,00-13,25=6,75 \mathrm{ccm}$ Bromidbromatlösung entspricht.

$1 \mathrm{ccm}$ verbrauchter Bromidbromatlösung entspricht $1,11 \mathrm{ccm}$ Äthylen ( $0^{\circ} \mathrm{C}, 760 \mathrm{~mm}$, Trockengas). Somit wurden also 7,49 ccm Äthylen (Normalbedingungen) gefunden. Auf $20^{\circ} \mathrm{C}, 750 \mathrm{~mm}$ und Feuchtgas umge-

- Ausgewählte oxydimetrische Titrationen unter Ersatz oder Einsparung von Jod. E. M e r ck, Darmstadt, S. 9 . rechnet, ergibt sich ein Gehalt des Analysengases von $13,9 \%$.

Die Tabelle ermöglicht den Vergleich der nach beiden Methoden erhaltenen Resultate aus jeweils gleichen Gemischen.

$\begin{array}{cc}\text { Jodometrisch } & \text { Mit Chloramin } \\ 1,68 & 1,69 \\ 3,22 & 3,21 \\ \mathbf{4 , 6 3} & \mathbf{4 , 5 3} \\ 6,57 & 6,58 \\ 7,80 & 7,80 \\ 10,56 & 10,53\end{array}$

Gefundene ccm Äthylen in $60 \mathrm{ccm}$ analysierten Gases.

Verwendete Lösungen

a) 0,1-n. Kaliumbromidbromat ${ }^{\circ}: 2,7835 \mathrm{~g} \mathrm{KBrO}_{3}$ und $12 \mathrm{~g} \mathrm{KBr}$ werden zu $1000 \mathrm{ccm}$ in Wasser gelöst.

b) 0,1-n. arsenige Säure ${ }^{7}$ 4,948 g $\mathrm{As}_{2} \mathrm{O}_{3}$ reinst werden in $40 \mathrm{ccm} n$-Natronlauge gelöst; die Lösung wird mit $40 \mathrm{ccm} n$-Salzsäure versetzt und mit Wasser auf $1000 \mathrm{ccm}$ aufgefüllt.

c) 0,1- $n$. Chloramin: $14,5 \mathrm{~g}$ Chlorina Heyden werden auf $1000 \mathrm{ccm}$ Wasser aufgefüllt.

7 F.Ḧ̈lzl, Anleitung zur Maßanalyse, Seite 114. Graz 1933.

\title{
Zur Frage der Biogenese von Terpenen: Dichloressigsäure als Polymerisationskatalysator
}

\author{
Von Theo LenNartz \\ Aus dem Chemotherapeutischen Forschungsinstitut „Georg-Speyer-Haus“, Frankfurt a.M. \\ (Z. Naturforschg. 1, 684-690 [1946]; eingegangen am 19. Aug. 1946)

\begin{abstract}
Arbeiten zur experimentellen Erhärtung der Vorstellungen über die Biogenese von
\end{abstract} \\ Terpenen aus den Hemiterpenalkoholen Dimethylvinylcarbinol oder Prenol führten zur \\ Auffindung der Dichloressigsäure als eines aliphatischen Polymerisationsbeschleunigers \\ sowohl dieser beiden Alkohole als auch des ihnen zugrunde liegenden Dienkohlen- \\ wasserstoffs, des Isoprens. Die Ergebnisse mit dieser einfachen Carbonsäure regen zur \\ Prüfung natürlicher organischer Säuren als Fermentmodelle an.
}

$\mathrm{U}^{\prime \prime}$ $\mathrm{m}$ zwei Moleküle Isopren offenkettig aneinanderzulagern, sind nur wenige Verfahren bekannt. Bei der Reduktion des Isoprens mit Kalium in Alkohol wurde ein Gemisch von Dimethyl-octadien-Kohlenwasserstoffen erhalten $(\mathrm{I})^{1}$. Läßt man auf Isopren Essigsäure in Gegenwart von Schwefelsäure einwirken, so wird zu einem relativ großen Teil Essigsäure in 1,4-Stellung an

1 Th. Midgley jr., A. L. Henne, J. Amer. chem. Soc. 51, 1293, 1294 [1929]; K. Z i e g ler u. H. Kleine r, Liehigs Ann. Chem. 473, 57 [1929].
Isopren angelagert unter Entstehung von Prenylacetat $^{2}$ (durch Verseifung daraus Prenol) und gleichzeitig Geranylacetat 3, 2 (daraus Geraniol) gebildet (II).

Mit Eisessig-Schwefelsäure konnte aus Isopren auch Farnesol $\left(\mathrm{C}_{15} \mathrm{H}_{26} \mathrm{O}\right)$, das Anlagerungsprodukt von Essigsäure an trimeres Isopren, gewonnen und als

2 Th. Lennartz. Ber. dtsch. chem. Ges. 76, 831 [1943].

3 Th. Wa g n e r-J a u r e g g, Liebigs Ann. Chem. 496. 52 [1932]. 


$$
\begin{aligned}
& \begin{array}{llll}
\stackrel{\mathrm{CH}_{3}}{\mathrm{I}} & \mathrm{CH}_{2}: \dot{\mathrm{C}} \cdot \mathrm{CH}: \mathrm{CH}_{2} \stackrel{\mathrm{K}, \mathrm{H}}{\longrightarrow} \mathrm{CH}_{3} \cdot \dot{\mathrm{C}}: \mathrm{CH} \cdot \mathrm{CH}_{3}, & \mathrm{CH}_{3} \cdot \dot{\mathrm{C}: \mathrm{CH}} \cdot \mathrm{CH}_{2} \cdot \mathrm{CH}_{2} \cdot \dot{\mathrm{C}}: \mathrm{CH} \cdot \mathrm{CH}_{3}
\end{array} \\
& \begin{array}{cl}
\mathrm{CH}_{3} \cdot \mathrm{CH}: \dot{\mathrm{C}} \cdot \mathrm{CH} \cdot{ }_{2} \mathrm{CH}_{2} \cdot \dot{\mathrm{C}}: \mathrm{CH} \cdot \mathrm{CH}_{3} & \mathrm{CH}_{3}
\end{array}
\end{aligned}
$$

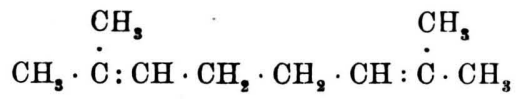

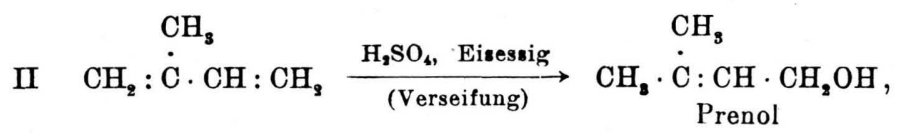

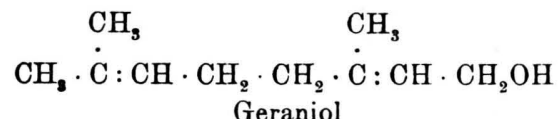

saures 3 -Nitro-phthalat charakterisiert werden ${ }^{2,4}$. Außerdem entstand ein aliphatischer Diterpenalkohol $\mathrm{C}_{20} \mathrm{H}_{34} \mathrm{O}^{2}$.

Bei diesen Reaktionen können die dimeren Stufen offenbar nur deshalb isoliert werden, weil der dimere Kohlenwasserstoff mit 3 Doppelbindungen intermediär im Falle I mit Wasserstoff, im Falle II mit Essigsäure, $\mathrm{H}+\ldots \mathrm{CH}_{3} \mathrm{COO}^{-}$, reagiert und so in das stabilere Diolefin übergeht. Fehlt bei der Polymerisation der Katalysator, z. B. bei der thermischen Polymerisation mit oder ohne Essigsäure ${ }^{5}$, so spielt sich eine Diensynthese im Sinne von Diels und Alder ab und es entsteht Dipren (III) oder Dipenten (IV). Die beiden anderen theoretisch möglichen, cyclischen Isoprendimeren V und VI sind bis heute noch nicht mit Sicherheit erhalten worden ${ }^{\circ}$.

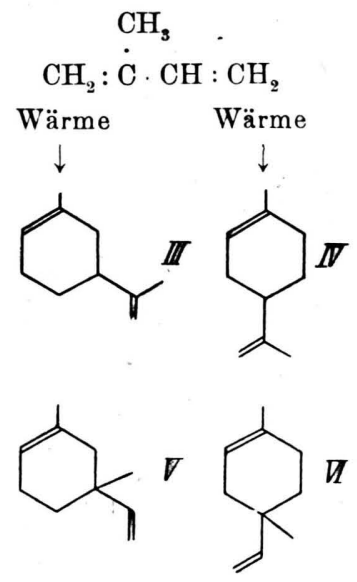

- Th. Lennartz, Ber. dtsch. chem. Ges. 76, 248 [1943]

s Vielleicht wirkt ein geringer Säurezusatz auf Isopren ähnlich stabilisierend wie bei Acrolein und seinen Homologen (H. Wagner, Schwed. P. $104640^{\circ}$ [1941]; De guss a, Frankfurt a. M., Chem. Zbl. 1942 II, 2315; 1943, I, 449).
Vor kurzem konnte gezeigt werden, daß aus Isopren durch Kontaktkatalyse an Clarit-Spezial, einem sauren Al-Silikat, ein aliphatisches Di-Isopren mit 3 Doppelbindungen entsteht ${ }^{7}$. Da dieses Di-Isopren neben Höherpolymeren und Isoprenharzen nur in verschwindender Menge aufgefunden wird, sehr leicht polymerisiert und sehr hygroskopisch ist, blieb es bei meinen Polymerisationsversuchen mit Clarit lange Zeit als Destillationsvorlauf, der in der Regel nur aus wenigen Tropfen bestand und schlechte (stark sauerstoffhaltige) Verbrennungen ergab, unbeachtet, und zu seiner Erkennung als aliphatisches Di-Isopren bedurfte es besonders sorgfältiger, Geduld fordernder Versuche. Die Konstitution des Di-Isoprens ist noch nicht genau erkannt. Es gilt zunächst noch, Reaktionsbedingungen herauszuarbeiten, unter welchen es in größerer Ausbeute anfällt.

Nach A. Harzer sind Triolefine vom Sdp. 158 bis $162^{\circ}$, Sdp. $1545^{\circ}$, aus Butadien und intern oder extern substituierten Butadienen, welche er aus Vorläufen der technischen Benzolerzeugung in Kokereien gewonnen hatte, auch durch 8-tägige Wärmepolymerisation bei $100^{\circ}$ erhältlich $^{*}$. Den Nachweis der Triolefine erhrachte er durch die Jodzahl und die Hydrierung mit Pd nach Skita.

Daß bei den aliphatiscleen Dimerisationsreaktionen, die von einer Addition anderer Elemente (I) oder Gruppen (II) zu stabilen Anlagerungsprodukten begleitet sind, unter dem katalytischen Einfluß der

- Siedenunktsunterschiede hei Ninrenfraktionen können durch einen wechselnden Gehalt an Wasser hedingt sein, da Dipren sehr hvgroskopisch ist, s. Th. Wagner-J a u regg u. Th. Lennartz, Ber. dtsch. chem. Ges. 76, 1163 [1943].

7 Th. Wagner-Jauregg u. Th. Lennartz, Ber. dtsch. chem. Ges. 76, 1161 [1943].

8 A. Harzer. J. f. Gashelenchtg., Wasserversorg 56. 750 [1913]; Chem. Zhl. 1913, II, 1088. - Die hier wiedergegehenen näheren Angaben sind brieflichen Privatmitteilungen von Hrn. Dr. Harzer, Freiberg i. S., entnommen. 


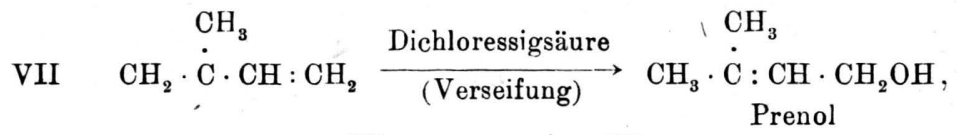

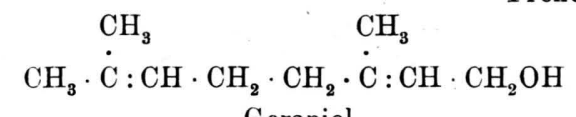

$$
\begin{aligned}
& \text { Geraniol } \\
& \text { VIII } \quad \stackrel{\mathrm{CH}_{3}}{\mathrm{CH}_{2}} \cdot \stackrel{\mathrm{CH}_{\mathrm{CH}}}{\mathrm{CH}_{2}}+\mathrm{CH}_{3} \cdot \stackrel{\mathrm{CH}_{3}}{\mathrm{CH}} \cdot \mathrm{CH}_{2} \cdot \mathrm{OCO} \cdot \mathrm{CH}^{\backslash \mathrm{Cl}}
\end{aligned}
$$

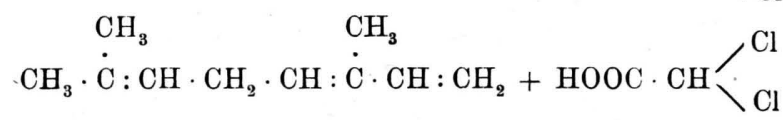

Schwefelsäure außer der normalen Kopf-SchwanzKopf-Schwanz-Verknüpfung der Isoprenmoleküle auch andere Aneinanderlagerungen vorkommen, die zu stärker verzweigten Molekülen führen, ist kürzlich beobachtet worden ${ }^{2}$. Es wird eine spätere Aufgabe sein, die Struktur dieser verzweigten Terpenalkohole aufzuklären.

In dem Bestreben, die Synthese von Terpenen aus Isopren bzw. den Hemiterpenalkoholen Prenol oder Dimethylvinylcarbinol mit Katalysatoren durchzuführen, welche als Fermentmodelle in Betracht gezogen werden können, um so die Annahme der Biogenese der Terpene aus den genannten Hemiterpenalkoholen ${ }^{9}$ experimentell auf eine festere Grundlage zu stellen, wurde nun gefunden, daß Dichloressigsäure sowohl Isopren als auch die beiden Hemiterpenalkohole in polymere, aliphatische Produkte umzuwandeln vermag.

Läßt man Dichloressigsäure und Isopren bei $20^{\circ}$ oder etwas höherer Temperatur mehrere Stunden aufeinander wirken und verseift das Reaktionsprodukt, so entsteht neben sehr wohlriechenden Kohlenwasserstoffen Prenol und Geraniol (VII).

Der Charakter der Anlagerung und der Polymerisation des Isoprens durch Dichloressigsäure entspricht dem mit Schwefelsäure in Eisessig bewirkten. Die Dichloressigsäure lagert sich ebenfalls ausschließlich in 1.4-Stellung an Isopren an, und zwar in der Weise, daß das Kation $\left.\mathrm{H}^{+}\right)$an das der Verzweigungsstelle benachbarte endständige C-Atom, das Anion $\left(\mathrm{CHCl}_{2} \mathrm{COO}^{-}\right)$an das entgegengesetzte Ende tritt. Wie aus der Isolierung von Geraniol aus der Terpenfraktion hervorgeht, ist die Verknüpfung der beiden Isopren-

9 Th. Lennartz, Ber. dtsch. chem. Ges. 76, 837 [1943]. moleküle durch normale Kopf-Schwanz-KopfSchwanz-Verkettung erfolgt. Ob daneben auch eine andere Verknüpfung zu stärker verzweigten Terpenalkoholen führte, konnte aus äußeren Gründen noch nicht einwandfrei festgestellt werden; da die Bildung der verzweigten Dimeren mengenmäßig viel geringer ist, sind größere Ansätze erforderlich.

Für die Entstehung der Terpenkohlenwasserstoffe, die auf ihre Konstitution noch nicht eingehender untersucht werden konnten, kann man an eine Reaktion zwischen Isopren und Prenyldichloracetat denken, und zwar derart, daß unter Wiederabspaltung von Dichloressigsäure ein Kohlenwasserstoff Dimethyloctatrien entsteht (VIII), der seinerseits Dichloressigsäure addiert, usw.

Die Anlagerungsprodukte der Dichloressigsäure an die monomere, dimere usw. Stufe des Isoprens, nämlich die Ester, sind als Zwischenstufen des gesamten Polymerisationsvorganges wohl nur deshalb faßbar, weil bei dem großen Aufgebot an Katalysator (Mol.-Verh. Isopren:Dichloressigsäure =1:1/2) die Konzentration an Isopren und, unabhängig davon, die Reaktionsfähigkeit mit zunehmender Kettenlänge schnell abnimmt. So kann auch der experimentelle Befund erklärt werden, daß mengenmäßig stets das Addukt der Säure an den monomeren Dienkohlenwasserstoff überwiegt und die höchsten Polymerisationsstufen, die hierbei erreicht werden, etwa $\left[\mathrm{C}_{5} \mathrm{H}_{8}\right]_{4-6}$ nur in sehr kleinen Anteilen vorhanden sind.

Der Chemismus der Polymerisation läßt sich unter diesen Gesichtspunkten in mehrere Teilreaktionen auflösen, im einfachsten Fall entsprechend dem allgemeinen Schema:

$$
\begin{aligned}
& \mathrm{A}+\mathrm{B}=\mathrm{AB} \\
& \mathrm{A}=\text { Isopren } \\
& \mathrm{AB}+\mathrm{A}=\mathrm{AA}+\mathrm{B} \\
& \mathrm{A}=\mathrm{K}=\mathrm{Katalysator} \text { (Dichlor- }
\end{aligned}
$$


Möglich wäre auch, daß aus Prenyldichloracetat Isopren in aktivierter, freier Form wieder abgelöst wird, welches eine Kettenreaktion auslöst und so zu den Polyisoprenkohlenwasserstoffen führt:

$$
\mathrm{AB} \longrightarrow \underset{\text { aktiv }}{\mathrm{A}}+\mathrm{B} \quad \underset{\text { aktiv }}{\mathrm{A}}+\mathrm{A} \longrightarrow \mathrm{AA}
$$

Die Primärreaktion, welche gleichzeitig die Polymerisation einleitet, ist offenbar in jedem Fall die Anlagerung von Dichloressigsäure an Isopren.
Während bei der Isoprenpolymerisation mit $\mathrm{H}_{2} \mathrm{SO}_{4}$ in Eisessig der Essigsäure lediglich die Funktion des Abfangens irgendeiner durch die Schwefelsäure bewirkten Polymerisationsstufe des Isoprens zugeschrieben werden kann, liegt in der Dichloressigsäure ein organischer Polymerisationsbeschleuniger vor, dessen Wirkung vermutlich als Hauptvalenzkatalyse im Sinne von W. Langenbeck aufzufassen ist und mit der Kondensation des Formaldehyds bei Gegenwart von Oxyketonen verglichen werden kann ${ }^{\mathbf{1 0}}$ :

$$
\begin{aligned}
& \mathrm{HCHO}+\mathrm{R} \cdot \mathrm{CH}(\mathrm{OH}) \cdot \mathrm{CO} \cdot \mathrm{R} \longrightarrow \stackrel{\mathrm{CH}_{2} \mathrm{OH}}{\stackrel{\dot{\mathrm{C}} \cdot \mathrm{CO} \cdot \mathrm{R}}{\dot{\mathrm{OH}}}} \\
& \mathrm{CH}_{2} \mathrm{OH} \\
& \mathrm{R} \cdot \dot{\mathrm{C}} \cdot \mathrm{CO} \cdot \mathrm{R}+\mathrm{HCHO} \longrightarrow \mathrm{CH}_{2}(\mathrm{OH}) \cdot \mathrm{CHO}+\mathrm{R} \cdot \mathrm{CH}(\mathrm{OH}) \cdot \mathrm{CO} \cdot \mathrm{R} \\
& \mathrm{OH}
\end{aligned}
$$

Polymerisationszwischenstufen sind bei der Dichloressigsäure-Polymerisation stabil und faßbar, wogegen die hypothetischen, die Polymerisation vermittelnden $\mathrm{H}_{2} \mathrm{SO}_{4}$-Addukte instabil sind und ihre niedrigmolekularen Glieder noch nicht isoliert worden sind.

Allgemeine Zusammenhänge zwischen beschleunigender Wirksamkeit und Konstitution des Katalysators lassen sich noch nicht erkennen. $\mathrm{Ob}$ die Aktivität ausschließlich von der H-Ionenkonzentration der Säure (Dissoziationskonstante der Dichloressigsäure $\left.k=5 \cdot 10^{-2}\right)^{11}$ abhängt, muß noch auf breiterer Basis überprüft werden.

Die Ausbeute an Prenol und Geraniol ist bei der Dichloressigsäure-Polymerisation des Isoprens nicht wesentlich größer als mit Eisessig-Schwefelsäure, aber die Reaktion verläuft im ganzen einheitlicher und es bildet sich dabei nicht jene Vielzahl von Stoffen (Terpenäther usw.) wie im anderen Fall. Überdies gestaltet sich das Arbeiten mit Dichloressigsäure einfacher und wirtschaftlicher, da man mit einem relativ geringen Materialaufwand auskommt.

Die Abtrennung der primären Alkohole geschah diesmal nicht über die Phthalester, sondern über die sauren 3-Nitro-phthalate. Dieses Verfahren erlaubt die Isolierung primärer Alkohole über ein

\footnotetext{
10 Naturwiss. 30, 30 [1942] ; s. a. Fermentforschg. 17, 387 [1944].

11 Dissoziationskonstanten nach Landolt-Börnstein, Physikal.-Chem. Tab., 5. Aufl., Springer.
}

kristallisiertes Derivat, welches in tiefsiedendem Petroläther schwer löslich ist, und bietet besonders bei der Untersuchung kleiner Materialmengen einige Vorteile.

Bei der Einwirkung von Dichloressigsäure auf $\mathrm{CH}_{3}$

Dimethylvinylcarbinol, $\mathrm{CH}_{3} \cdot \dot{\mathrm{C}} \cdot \mathrm{CH}: \mathrm{CH}_{2}$ (IX), $\mathrm{OH}$

während $5 \mathrm{Tgn}$. bei Zimmertemperatur bildete sich, wie mit Eisessig- $\mathrm{H}_{2} \mathrm{SO}_{4}{ }^{12}$, auf dem Umweg über die Dichloressigsäureester Prenol und Geraniol, neben Sesqui- und Diterpenkohlenwasserstoffen.

Aus Prenol war unter denselben Bedingungen nach 19 Tagen nur wenig eines Polymerisates vom Sdp. 100 bis $125^{\circ}$ entstanden. Mit 3-Nitro-phthalsäureanhydrid bildete sich daraus kein Ester. Auch der Geruch wies nur auf Kohlenwasserstoffe, nicht auf Terpenalkohole hin.

Das Geraniol kann man sich aus Dimethylvinylcarbinol über die Isoprenzwischenstufe entstanden vorstellen, an welche sich die Dichloressigsäure wie oben anlagert. Daß Prenol mit Dichloressigsäure nur sehr langsam reagierte, liegt wohl daran, daß der primäre Alkohol schwerer Wasser abspaltet als der tertiäre.

Die Tatsache, daß der tertiäre Hemiterpenalkohol Dimethylvinylcarbinol (IX) mit Dichloressigsäure, im Gegensatz zum Prenol, so leicht unter Terpenbildung

12 Th. Lennartz, Ber. dtsch. chem. Ges. 76, 836 [1943]. 


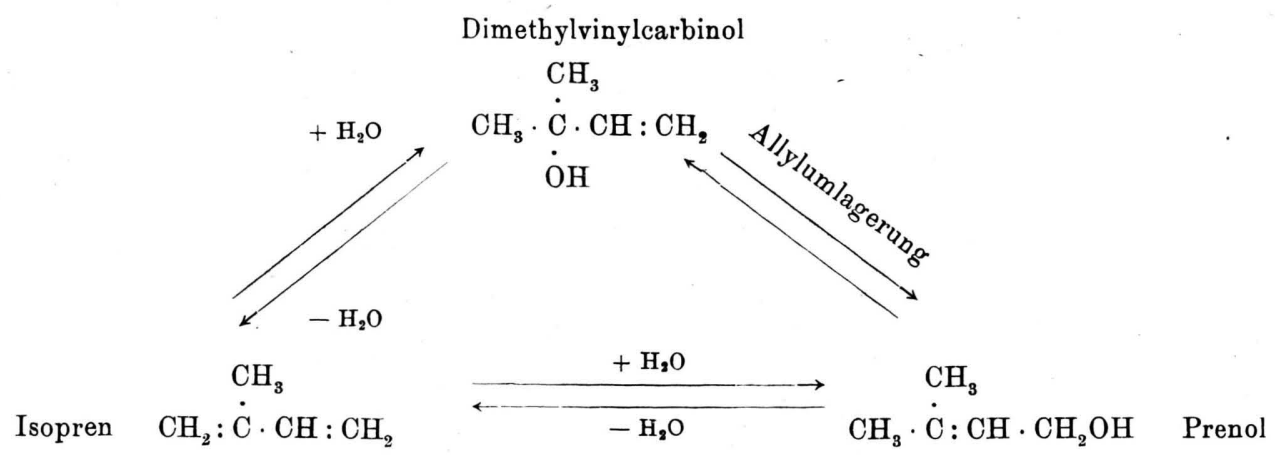

reagiert ${ }^{13}$, unterstreicht die mögliche Bedeutung dieses tertiären Alkohols für die Genesis der Terpene in der Natur. Daß neben dem Prenol, welches sich u. a. im Foeniculin (X) ${ }^{14}$ veräthert findet, auch das Dimethylvinylcarbinol in der Natur eine Rolle spielt, zeigt z. B. das Formelbild des Xanthyletins (XI) ${ }^{15}$. Hier liegt es am Benzolkern gebunden vor, teils direkt am Kohlenstoff, teils veräthert.<smiles>CC=Cc1ccc(OCC(C)C)cc1</smiles>

$X$<smiles>CC1(C)Oc2cc3c(cc2O1)OCCO3</smiles>

BI
Es ist kaum anzunehmen, daß die Natur zum Aufbau der Terpene nur von einem einzigen Baustein auszugehen vermag. Vielmehr ist zu erwarten, daß an der Synthese einer solch vielgestaltigen Klasse von Naturstoffen wie Terpenen und Terpenoiden mehrere, verwandte Bausteine beteiligt sind. Als Modellsubstanzen sind deshalb verschiedene Isopentan- bzw. Isopentenkörper in Erwägung zu ziehen, und zwar in erster Linie solche mit funktionellen Gruppen wie Alkohole, Aldehyde und Säuren. Die für den Aufbau aus $\beta$-Methyl-crotonaldehyd sprechenden Argumente sind von W.S andermann eingehend erörtert worden ${ }^{16}$. Die Synthese von Terpenen aus Säuren, z. B. Isoprencarhonsäure (XII) ${ }^{17}$, scheiterte bisher an der Schwierigkeit, unter physiologischen (oder wenigstens milden) Bedingungen $\mathrm{CO}_{2}$ abspalten zu können. In diesem Zusammenhang gehührt indes der Isolierung von Brenzterehinsäure (XIII) ${ }^{\mathbf{1 8}}$ als der 14- Dihydro-isoprencarbonsäure aus dem in Afrika als Pfeilgift benutzten Milchsaft von Calotropis procera (Asklepiadaceen) Beachtung.

$\begin{array}{cc}\mathrm{CH}_{3} & \mathrm{CH}_{3} \\ \mathrm{CII} & \dot{\mathrm{C}} \cdot \mathrm{CH}: \mathrm{CH} \cdot \mathrm{COOH} \\ \mathrm{CH}_{3} \cdot \dot{\mathrm{C}}: \mathrm{CH} \cdot \mathrm{CH}_{2} \cdot \mathrm{COOH}\end{array}$

13 Aus Dimethylvinylcarbinol (mit 20-proz. $\mathrm{H}_{2} \mathrm{SO}_{4}$ ) konnten auch Faw orski und Lebedeva Terpenalkohole gewinnen, Bull. Soc. chim. France, Mém. [5] 6, 1347 [1939].
Für die biologische Entstehung von Terpenen aus einem Hemiterpenalkohol seien die nahen reaktiven Beziehungen zwischen Dimethylvinylcarbinol und Prenol bzw. Isopren hervorgehoben. Der stabilste und reaktionsträgste Stoff ist das Prenol. Wenn auch Isopren noch nicht aus Pflanzen isoliert worden ist, so ist seine Bedeutung als intermediäre $\mathrm{Zw}$ wischenstufe doch nicht von der Hand zu weisen.

Die intermediäre Rolle des Isoprens für die natürliche Entstehung von Terpenen entspricht wahrscheinlich derjenigen des Formaldehyds, welchen die grüne Pflanze (nach den Annahmen von A. v. B a e y e r, R. Wills tät t e r, ferner A. St o ll, O. Wa r bu r g) als erstes Assimilationsprodukt aus $\mathrm{CO}_{2}$ und Sonnenlichtenergie unter Beteiligung des Chlorophylls bildet; vermutlich werden aus dem reaktionsfähigen Formaldehyd durch sofortige Weiterkondensation Kohlenhydrate, Fette und, nach Einbau von Stickstoff, Eiweißstoffe aufgebaut.

Bei dem heutigen Stand der Hypothesen üher die Biogenese der Terpene erscheint mir vor allem eine intensivere experimentelle Bearbeitung der schwehenden Fragen vonnöten. Wichtig ist dahei das Studium von Katalysatoren. Nach den geschilderten Ergebnissen mit Dichloressigsäure und Isopren oder Dimethylvinylcarbinol erhebt sich die Frage, ob vielleicht ganz einfach gebaute organische Säuren als Biokatalysatoren wirksam sein könnten. Hier wäre es interessant, einige in Pflanzen vorkommende Säuren, etwa die weit verhreitete Oxalsäure, deren Dissoziationskonstante größenordnungsmäßig derienigen der Dichloressigsäure entspricht, auf ihr Vermögen als Fermentmodell zu untersuchen.

Die katalvtische Wirkung der Srhwefelsäure in Eisessig auf die Isoprenpolymerisation läßt sich in gewissem Umfang auch mit starken organischen Säuren, wie p-Toluolsulfonsäure, erzielen. Aus solchen Ansätzen konnten ebenfalls über die Essigsäureester Pre-

14 F. Snäth u. J. B r u ck, Ber. dtsch. chem. Ges. 71, 2709 「19381.

15 E. S p ät h u. W. M o čni k, Ber. dtsch. chem. Ges. 70, 2276 [1937].

16 Wien. Chem. Ztg. 45, 49 [1942].

17 Th. Lennartz, Ber. dtsch. chem. Ges. 76, 1006 [1943].

18 G. Hesse, H. Eilhrecht u. F. Reicheneder, Liebings Ann. Chem. 546, 233 [1940]. 
nol und Geraniol neben viel Kohlenwasserstoff isoliert werden.

\section{Beschreibung der Versuche ${ }^{19}$}

Das von der I.G. Farbenindustrie AG., Werk Ludwigshafen a. Rh., in dankenswerter Weise überlassene Isopren wurde vom Stabilisator abdestilliert und mit Natrium über eine $1 \mathrm{~m}$ hohe Wi d m e r-Spirale fraktioniert. Verwandt wurde der von 31 bis $34^{\circ}$ siedende Anteil.

\section{Isoprenpolymerisation mit Dichloressigsäure}

Eine Lösung von $128 \mathrm{~g}$ Dichloressigsäure (1 Mol) und $1,4 \mathrm{~g}$ Hydrochinon in $136 \mathrm{~g}$ Isopren (2 Mol) wurden 8 Stdn. auf $40^{\circ}$ erwärmt. Das braungefärbte Reaktionsgut wurde in Äther mit 2- $n$. $\mathrm{NaOH}$, dann mit Wasser neutral gewaschen und über $\mathrm{Na}_{2} \mathrm{SO}_{4}$ getrocknet. Ausb.: $176 \mathrm{~g}$ von angenehmem, an Aprikosen erinnerndem Geruch. $120 \mathrm{~g}$ dieses Rohproduktes wurden mit $350 \mathrm{~g}$ 20-proz. methanol. KOH 50 Min. auf dem Dampfbad verseift, der Methylalkohol etwa halb abgedampft, mit viel Wasser verdünnt und 4-mal mit Petroläther (bis $60^{\circ}$ ) ausgeschüttelt, der Petroläther mit gesättigter Natriumchlorid-Lösung gewaschen, über $\mathrm{Na}_{2} \mathrm{SO}_{4}$ getrocknet und abgedampft. Der Rückstand (58 g), der halogenfrei war (Beilstein), wurde im WidmerKolben fraktioniert.

Prenol: Die nach 3-maliger Destillation (5 g) ${ }^{20}$ gewonnene Fraktion vom Sdp.13 48 bis 49,5 ${ }^{\circ}$, Sdp.746 137 bis $139^{\circ}$ bestand aus analysenreinem 2-Methyl-buten(2)-ol-(4) und unterschied sich auch im Geruch nicht von einem früher durch katalytische Anlagerung von Essigsäure an Isopren gewonnenen Präparat ${ }^{2}$.

$$
n_{\mathrm{D}}^{19} 1,4439, \quad d_{4}^{19} 0,8679 \text {. }
$$

$\mathrm{C}_{\mathbf{b}} \mathrm{H}_{10} \mathrm{O}(86,08)$. Ber. C 69,70, H 11,70, Mol.-Refr. 26,36. Gef. C 69,59, H 11,61, Mol.-Refr. 26,34. $69,55, \quad 11,69$.

Phenylurethan: Schmp. $64,5^{\circ}$ (Kofler), Mischprobe.

Auch aus den Vor- und Nachläufen der PrenolFraktionierung konnten nur Phenylurethane vom Schmp. $64^{\circ}$ (Kofler) erhalten werden; es wurden also keine Alkohole, die durch anders geartete Addition von Dichloressigsäure an Isopren denkbar wären, aufgefunden.

Geraniol: Aus den zwischen 90 bis $125^{\circ} / 14 \mathrm{~mm}$ siedenden Anteilen, die eine beträchtliche Menge Kohlenwasserstoffe enthielten, wurden die primären Alkohole über die sauren 3-Nitro-phthalate abgetrennt. $\mathrm{Zu}$ diesem $Z$ wecke ließ man die betreffende Fraktion mit einer Lösung von 3-Nitro-phthalsäureanhydrid (18 g) in der erforderlichen Menge absoluten Pyridins (80 ccm) 3 Tage bei Zimmertemperatur stehen, nahm mit Äther auf, säuerte mit verd. $\mathrm{HCl}$ an, wusch mit $\mathrm{H}_{2} \mathrm{O}$ neutral und trocknete über $\mathrm{Na}_{2} \mathrm{SO}_{4}$. Aus dem Ätherrückstand fielen beim Verreiben mit Petroläther

19 Alle Schmelzpunkte unkorrigiert.

20 Die Verseifung zu Prenol gab stets höchstens 50\% Ausbeute. (bis $40^{\circ}$ ) Blättchen aus, die auf einer engporigen Glasfritte abgesaugt, mit Petroläther $\left(40^{\circ}\right)$ gewaschen und mit überschüssiger 10-proz. methanol. $\mathrm{KOH}$ 30 Min. verseift wurden. Nach geringem Wasserzusatz wurde $1 / 3$ des Methanols verdampft, mit Wasser stark verdünnt und 5-mal mit Petroläther (bis $60^{\circ}$ ) ausgeschüttelt, die Petroläther-Lösung mit NaCl-Lösung gewaschen, über $\mathrm{Na}_{2} \mathrm{SO}_{4}$ getrocknet. Die Hauptmenge des Rückstandes ging im Widmer-Kolben bei 110 bis $115^{\circ} / 13 \mathrm{~mm}$ über. Sein Geruch schien zarter als der des natürlichen Geraniols.

$$
\begin{gathered}
n_{\mathrm{D}}^{19,5} 1,4731, \quad d_{4}^{19,5} 0,8869 . \\
\mathrm{C}_{10} \mathrm{H}_{18} \mathrm{O}(154,14) \text {. Ber. C 77,85, H 11,77, Mol.-Refr. F2 48,97. } \\
\text { Gef. C } 77,78, \mathrm{H} 11,92, \quad, \quad " \quad \begin{array}{r}
48,78, \\
48,79 .
\end{array}
\end{gathered}
$$

3.5-Dinitrobenzoat: Aus $126 \mathrm{mg}$ Alkohol, $240 \mathrm{mg}$ Dinitrobenzoylchlorid in 2,5 ccm Benzol und 0,25 $\mathrm{ccm}$ Pyridin nach 2-tägigem Aufbewahren bei Zimmertem- peratur. Schmp. 59,5 bis $60,5^{\circ}$ (K ofler), aus Petroläther $60^{\circ}$. Mischschmp. mit einem Dinitrobenzoat vom Schmp. 60,5 bis $62^{\circ}$ aus natürlichem Geraniol: 60 bis $61^{\circ}$ (K o f l e r).

Das gleiche Resultat wurde erzielt, wenn man die Dichloressigsäure 48 Stdn. bei Zimmertemperatur auf Isopren einwirken ließ.

\section{Prenol und Geraniol aus Dimethylvinylcarbinol und Dichloressigsäure}

Ein Gemisch von $14 \mathrm{~g}$ Dimethylvinylcarbinol und $18,7 \mathrm{~g}$ Dichloressigsäure (Molverhältnis 1:1) wurde nach 5-tägigem Stehenlassen bei Raumtemperatur in gesättigte Kochsalzlösung gegossen, mit 2-n. $\mathrm{Na}_{2} \mathrm{CO}_{3}$, dann mit Kochsalzlösung neutral gewaschen und über $\mathrm{Na}_{2} \mathrm{SO}_{4}$ getrocknet. Im Vak. wurden 2,6 g Dimethylvinylcarbinol entfernt. Das übrige verseifte man mit 10-proz. methanol. $\mathrm{KOH}$ wie üblich und trennte die entstandenen Produkte durch Destillation im Widmer - Kolben:

Sdp.13 49 bis $51^{\circ}, 1,2$ g Prenol, Phenylurethan Schmp. $64^{\circ}$ (K o fle r), Sdp.11 $100-108-113^{\circ}, 0,74 \mathrm{~g}$ Geraniol, 3-Nitrophthalestersäure, Schmp. 107 bis $108^{\circ}$, Sdp.11 119 bis $127^{\circ}, 0,6 \mathrm{~g}$, Rüekstand: $\quad 3,5 \mathrm{~g}$.

\section{Isoprenpolymerisation mit p-Toluolsulfonsäure in Eisessig}

$120 \mathrm{~g}$ Isopren wurden mit einer Lösung, von $25 \mathrm{~g}$ pToluolsulfonsäure puriss. in $500 \mathrm{~g}$ Eisessig (98 bis $100 \%$ ) vermischt (5\%, bezogen auf Eisessig) und $48 \mathrm{Stdn}$. bei etwa $18^{\circ}$ sich selbst überlassen. Aus zwei derartigen Ansätzen wurden nach der oben beschriebenen Aufarbeitung $268 \mathrm{~g}$ Rohprodukt gewonnen und daraus $80 \mathrm{~g}$ als ein in Methanol schwerlöslicher Anteil abgetrennt. Vom übrigen gingen $145 \mathrm{~g}$ zwischen 80 und $125^{\circ} / 12 \mathrm{~mm}$ über. Diese wurden mit $16 \mathrm{~g}$ Natrium in 
$500 \mathrm{ccm}$ absol. Methanol zur Verseifung $3^{1} / 2$ Stdn. bei Zimmertemperatur stehen gelassen, dann mit Wasser verdünnt und mit Essigsäure schwach angesäuert. Mit Äther konnten der wäßr. Phase $124 \mathrm{~g}$ des Reaktionsgutes entzogen werden, die mit $130 \mathrm{~g}$ Phthalsäureanhydrid in $500 \mathrm{ccm}$ Pyridin in üblicher Weise zur Abtrennung der primären Alkohole behandelt wurden. Aus $21 \mathrm{~g}$ der rohen Alkohole ließen sich $6 \mathrm{~g}$ Geraniol vom Sdp.14 $100-112-115^{\circ}$ herausdestillieren.

$$
n_{\mathrm{D}}^{20} 1,4726, \quad d_{4}^{20} 0,8858 .
$$

Mol.-Refr. $\mathrm{C}_{10} \mathrm{H}_{18} \mathrm{O}$ F2, ber. 48,97, gef. 48,78.

3.5-Dinitrobenzoat: Schmp. 60 bis $61^{\circ}$ (K of le r). Die Mischprobe mit dem Dinitrobenzoat aus natürlichem Geraniol schmolz bei 60 bis $61,5^{\circ}$.

Aus einem Ansatz von $30 \mathrm{~g}$ Isopren mit 2,5 g $p$ Toluolsulfonsäure in $125 \mathrm{~g}$ Eisessig (98 bis 100\%, p.a.), also $2 \%$ des Eisessigs, der $52 \mathrm{Stdn}$. bei $18^{\circ}$ gestanden hatte, wurden $7,3 \mathrm{~g}$ Prenylacetat vom Sdp.11 46 bis $48^{\circ}$ gewonnen. Diese Fraktion, mit methanol. $\mathrm{KOH}$ verseift, ergab $3 \mathrm{~g}$ Prenol vom Sdp.751 138 bis $140^{\circ}$.

$$
n_{\mathrm{D}}^{21} 1,4450 . \quad d_{4}^{21} 0,8691 .
$$

Mol.-Refr. $\mathrm{C}_{5} \mathrm{H}_{10} \mathrm{O}$ F1, ber. 26,36, gef. 26,36 .

Phenylurethan: Schmp. $64^{\circ}$ (Kofler), Mischprobe ebenso.

Die nachfolgende Übersicht enthält die Ausbeuten aus je $30 \mathrm{~g}$ Isopren in $125 \mathrm{~g}$ Eisessig (98 bis 100proz.) mit variierten Katalysatorkonzentrationen (bezogen

\begin{tabular}{|c|c|c|c|c|c|}
\hline \multirow{2}{*}{$\begin{array}{l}p \text {-Toluol- } \\
\text { sulfon- } \\
\text { säurekon- } \\
\text { zentration } \\
\text { in } \%\end{array}$} & \multirow{2}{*}{$\begin{array}{l}\text { Reakt.- } \\
\text { dauer } \\
\text { in Stdn. }\end{array}$} & \multirow{2}{*}{ 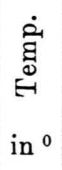 } & \multirow{2}{*}{$\begin{array}{l}\text { Gesamt- } \\
\text { ausb. roh } \\
\text { in } g\end{array}$} & \multicolumn{2}{|c|}{$\operatorname{Sdp}_{\cdot_{11}}$} \\
\hline & & & & $\begin{array}{c}45-50^{\circ} \\
\mathrm{g}\end{array}$ & $\begin{array}{c}100-120^{\circ} \\
\mathrm{g}\end{array}$ \\
\hline 0,5 & 112 & 18 & 13 & 6,1 & 4,7 \\
\hline 1 & 18,5 & 20 & 10 & 3,5 & 3 \\
\hline 2 & 52 & 18 & 18 & 7,3 & $8, \tilde{5}$ \\
\hline 4 & 50 & 18 & 30 & 5,9 & $14^{\prime}$ \\
\hline 5 & 48 & 18 & 33 & 4,4 & 15,9 \\
\hline 6 & 40 & 18 & 35 & 3,2 & 16,9 \\
\hline
\end{tabular}
auf Eisessig):

Durch Steigerung der $p$-Toluolsulfonsäurekonzentration kann also die Gesamtausbeute an Polymerisat erhöht werden, doch nimmt die Ausbeute an Prenylacetat parallel damit wesentlich ab, zugunsten der höher siedenden Fraktionen, die dann zum größten Teil aus Kohlenwasserstoffen bestehen.

Bei Versuchen mit $1 \%$ Trichloressigsäure $(k=$ $\left.2 \cdot 10^{-1 / 18^{\circ}}\right)$ oder $1 \%$ Pikrinsäure $\left(k=1,6 \cdot 10^{-1} / 25^{\circ}\right)$ in Eisessig konnte keine Anlagerung oder Polymerisation beobachtet werden.

Hr. Hans Lieber hat mich bei der Ausführung der Versuche fleißig unterstützt.

\title{
Weitere Röntgeninterferenzen bei Keratinen ${ }^{1}$
}

\author{
Von Helmut $Z_{\text {AhN }}$
}

\author{
Aus dem Institut für Textilchemie in Badenweiler
}

(Z. Naturforschg. 1, 690-691 [1946]; eingegangen am 24. Oktober 1946)

\begin{abstract}
Keratin-Röntgenogramme enthalten verwaschene D e b y e - S c h e r r e r - Ringe, deren Schwärzungsmaxima Abständen von $4 \div 4,6,2,1 \div 2,3,1,5 \div 1,6$ und $1,1 \div 1,2 \AA$ entsprechen.
\end{abstract}

$\mathrm{D}$ ie Röntgenogramme von Keratinen enthalten im normalen Winkelbereich außer den von W. T. Astbury und A. Street ${ }^{2}$ festgestellten Interferenzen noch einige verwaschene DebyeScherrer-Ringe, welche eigenartigerweise bisher übersehen worden sind. Sie entsprechen Netzebenenabständen von $4 \div 4,6^{3}, 2,1 \div 2,3,1,5 \div 1,6$ und 1,1 - 1,2 A. Ein Teil dieser Interferenzen wird bei verhältnismäßig großen Winkeln abgebeugt und entzieht sich dem Nachweis in der bei Faserauf-

1 20. Mitteilung aus der Reihe ,Struktur und Reaktionsfähigkeit der Keratine", vorige Mitteilung vergl. E. El öd u. H. Z a h n, Melliand Textilber. 27, 68 [1946].

2 Philos. Trans. Roy. Soc. London Ser. A 230, 75 [1931]. nahmen üblichen L a u e-Kammer, während eine zylindrische Aufnahmekamera günstigere Intensitätsverhältnisse bietet. Die Interferenzen treten bei verschiedenen Keratinsorten, wie Wolle, Roßhaaren, Stachelschweinkiel, auf, und zwar ist es in erster Näherung gleichgültig, ob die Proben im ungedehnten ( $\alpha$-Keratin), im gedehnten ( $\beta$-Keratin) oder denaturierten Zustande (d-Keratin) vorliegen. Bezüglich der Anisotropieverhältnisse fällt auf, daß die Interferenzen im Äquator etwas stärker ausgeprägt sind, man hat sie jedoch als Inter-

3 R. B. Cor e y u. R. W. G. W y c k of f (Journ. Biol. Chem. 114, 407 [1936]) verzeichneten ebenfalls einen amorphen Halo auf dem Äquator bei $4,6 \AA$. 